\title{
Cyber Rabbit: Adventures in Cyberland
}

\author{
Batuhan Bintas \\ Cyber Rabbit Studios \\ London, UK \\ info@batuhanbintas.com
}

\begin{abstract}
We live in a very interesting age where science fiction is becoming science fact. It has been only 20 years since the World Wide Web connected all of us, only 10 years since smart devices entered our pockets, and today we are experiencing the rise of the Artificial Intelligence. As if this was not exciting enough, we are creating Cyber Worlds that we can access by using Augmented- (AR), Mixed- (MR), Virtual- (VR) Reality tools. The age we live in requires not only the creation of more sophisticated tools, but also understanding the responsibility that comes with these creations. Cyber Rabbit is an emerging decentralised Extended Reality studio that explores the role of technology in our collective evolution. The organisation's first product "Adventures in Cyberland" is a book that simplifies the communication language of these technological developments and informs the reader through storytelling with XR technologies. The book is a platform both for children and adults to learn about our growing civilisation and raises awareness about society's most recent topics.
\end{abstract}

New forms of experience. Extended Reality. Following the Cyber Rabbit.

\section{INTRODUCTION}

Lewis Carroll's Alice's Adventures in Wonderland is one of the brilliant cult stories that people from all age groups enjoy reading. What makes Alice's Adventures in Wonderland really special is that the story not only entertains the reader, it makes us curious about fundamental questions - from the origins of reality to how our perception can be tricking us.

The story gained popularity during the hippy movement of the 1960s and since then there have been many different forms of Alice in Wonderland. From movies to different illustrated books, not to mention numerous references to it in popular culture, Alice has become a symbol of curious minds.

Yet until now, we always read the story of Alice or experienced Wonderland from her perspective. What would we do if we were given the opportunity to follow the White Rabbit of the story ourselves?

\subsection{Adventures in Cyberland}

Adventures in Cyberland is an illustrated book that invites the reader to explore what is happening in a cyber-reality parallel to our own, hidden inside the pages of the book. Each page of the book is a painting that is a portal, gateway into the Cyberland.

The book applies 'context engineering' (a term that will be discussed in more detail later in this paper) to the traditional way of reading a story. Instead of following the story of Alice by reading the book, the audience becomes the 'Aliceer'; they are invited to follow a Cyber Rabbit and enter directly into the world of the story hidden on each page through the Extended Reality $(\mathrm{XR})^{*}$ experiences activated by a "Cyber Mushroom". We choose to use the word Extended Reality (XR) here to combine Augmented Reality (AR), Mixed Reality (MR) and Virtual Reality (VR); XR also gives the option for the user to define the reality they experience by defining the ' $X$ '.

Cyber Mushroom is the smart device app that is designed to take the 'Aliceers' to an Adventure in Cyberland. The app combines AR, MR and VR technologies, allowing the audience to digitally interact with every page and with the stories of the book. The audience can choose whether to interact with illustrations and characters by augmenting the reality of the page, or fall into the story on the chosen page through virtual reality. 
In Cyberland, our Aliceers go through stories that explore counter-culture ideas, which challenge the status quo. For instance, these explore the decentralisation of society, creating a new value system together via the use of cryptocurrencies. Therefore Adventures in Cyberland aims to use XR to explore ideas related to the mysteries of the mind, the creation of Als, and the future of our collective evolution.

\section{CYBER RABBIT}

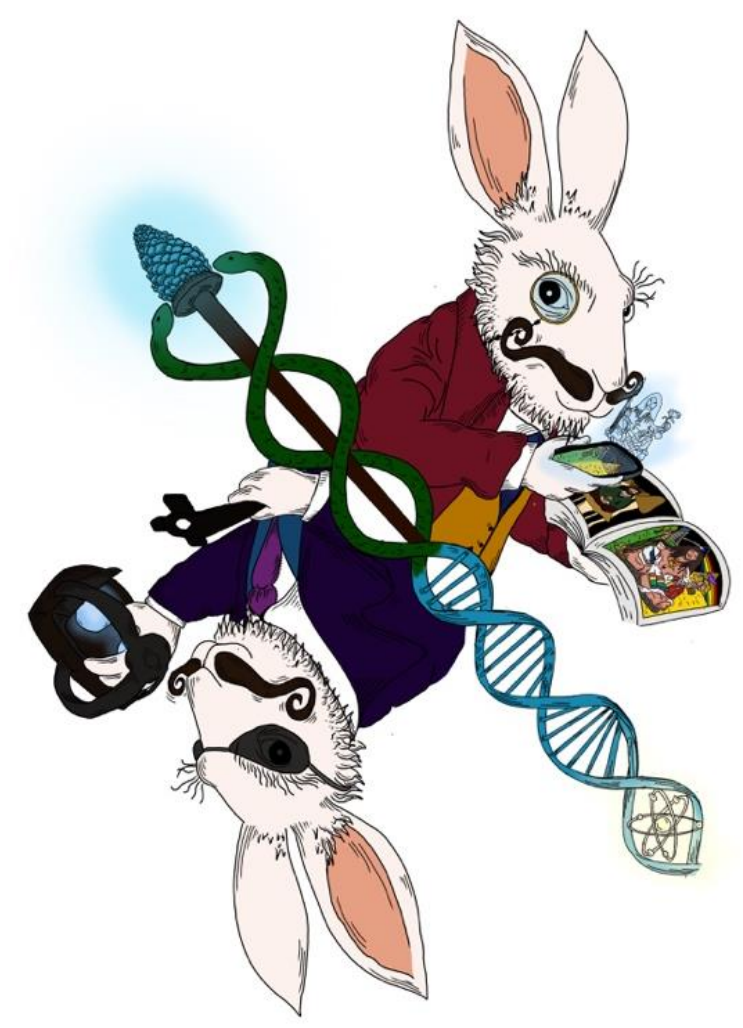

Figure 1: Cyber Rabbit illustration.

\subsection{Vision}

We live in a time where we experience science fiction stories that we grew up with becoming 'science fact'. Therefore we may constantly wonder what that future will be like. Yet how can we talk about brighter futures when our young and fresh minds are being programmed in an environment that normalises hatred and fear?

The contents and education systems that our younger generations are growing up with do not empower the creative potential of the individuals; instead they force-feed young minds with ideas that separate our community through sexism, racism, classes, violence and war. In a nutshell, children are being dumbed down and brainwashed to accept and even serve the rapacious system of artificial scarcity, unending exploitation, and incessant war. Adults of older generations have also been exposed to these influences to an extent.

In a world where science fiction is becoming 'science fact', the younger generation needs to grow through fiction that empowers their creative potential, encouraging them to follow their dreams. Fiction for children must also be simplified to be understandable for children. On the other hand, adults also need a platform where they can reconnect with their childlike minds and re-spark the fire of curiosity in searching for magic and wonder in our experiences of reality. This is so we can reclaim our individual potential fully and break out of the illusions and separations that are created by the current 'operating system' in which we live. Then we can start building our future collectively on a stable foundation.

Cyber Rabbit's vision is to allow the creative potential of individuals to flourish and plant seeds of wonder and curiosity to explore the magic and mystery of our reality, by using the potential of new $X R$ technologies to empowering the imagination through cyberspace.

\subsection{Mission}

Through passion, fun and curiosity, we work on changing the way our society approaches this experience of reality that we are going through collectively, by daring to ignite the mind, inspire the spirit and penetrate the heart with wonder seeds that spark curiosities for new explorations.

Cyber Rabbit is a decentralised XR studio that is on a mission to provide experiences that extend the possible realities in reach; a decentralised educational XR playground where people can choose what contents our younger minds need to be growing up with and what needs to be addressed in our society.

Our belief in providing decentralised organisation allows us to fully understand and reflect the demands of our growing civilisation, taking the power of decision-making from a small group of individuals and giving it to people of all ages, backgrounds and races. This gives this platform the chance to provide children's content that is created collectively, so that different parts of society can relate to one another.

Soon, we are planning to release ICO (Initial Coin Offering) tokens that can be used by people to suggest characters and story ideas to Cyber Rabbit and collectively fund them to bring them into existence in the Educational XR Platform. It will be possible for ICO tokens to be bought as well as earned by contributing towards building this paradigm collectively. Thus, both children and 
adults will be able to go into our platform, build the characters and stories, and earn tokens.

We use the potential of the cutting edge $X R$ technologies in order to have a deeper impact on the human psyche and allow the experiencer to become immersed in the story, and become part of it. Our goal is to empower the imagination through cyberspace.

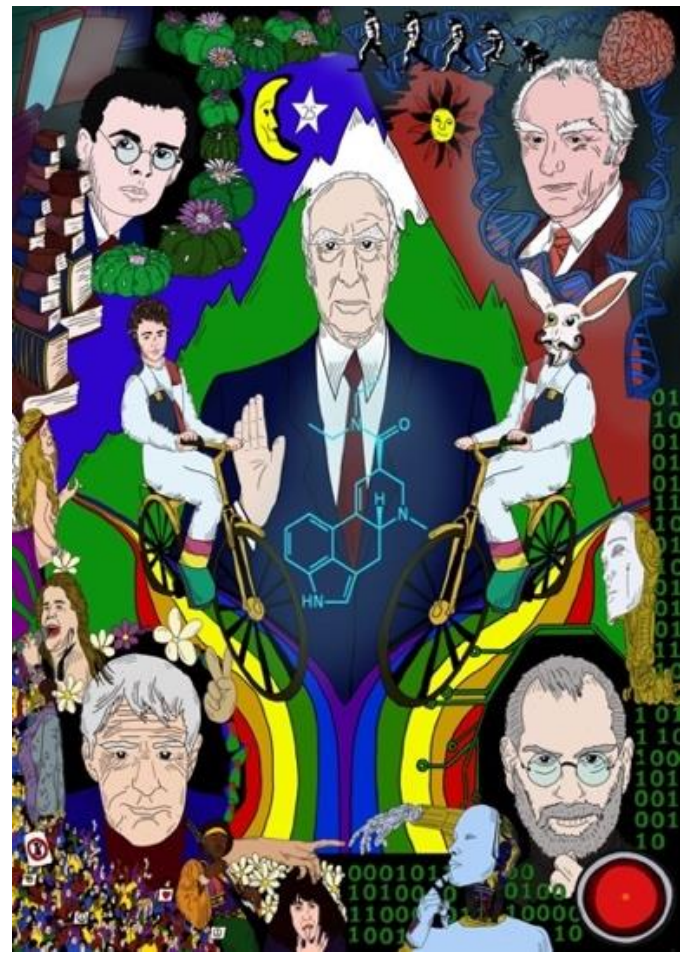

Figure 2: Bicycle day illustration.

\section{ADVENTURES IN CYBERLAND}

\subsection{Story}

We live in a time where the line between science fiction and scientific fact is becoming blurred. Adventures in Cyberland is an experience that aims on sharpening that blur by raising awareness on leading topics that are becoming a very important part of our society. Following the Cyber Rabbit will lead the audience into Cyberland where on each page they will meet characters and have adventures that explore topics such as:

- Artificial Intelligence

- The Internet

- Decentralised society

- Cryptocurrencies

- Global basic income

- Future of our planet

- Expansion of reachable realities through XR technologies

- Virtual Empathy tools

- Cyberdelics
- Future of evolution

- Empowerment of the creative potential of the individual

- Exploring the mind

- Altered States of Consciousness

- Claiming the new information technologies

- Decentralised media

Adventures in Cyberland is an experience that is present in the physical reality in book form, which has stories on each page that are told through poetic, visionary and symbolic paintings/illustrations that are the gateways into the experiences in Cyberland.

\subsection{Cyber Mushroom}

Cyber Mushroom is the tool that opens these gateways between our reality and Cyberland and allows our 'Aliceers' to step through them into the Adventures in Cyberland.

The mobile app combines AR, MR and VR technologies in order to activate the Extended Reality experiences that are embedded on each painting on each page. The audience can choose whether to interact with illustrations and characters by Augmenting the reality of the page or fall into the story of the chosen page by turning the experience into Virtual Reality. See some examples in Figures 3-5.

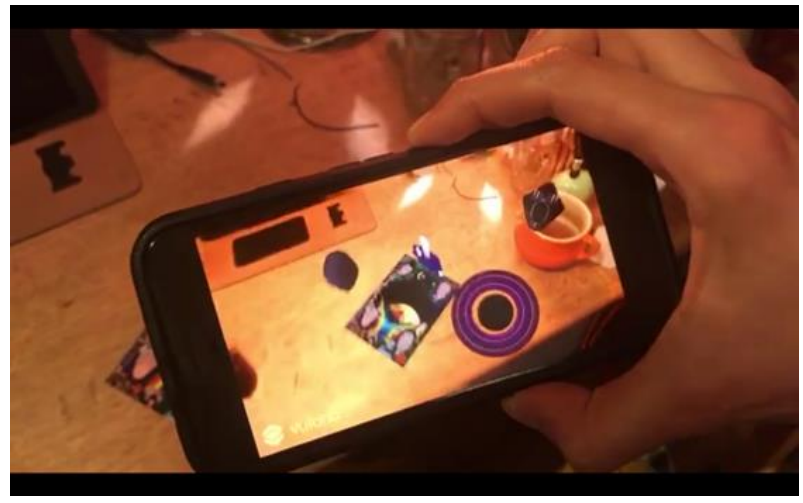

Figure 3: AR-augmenting the current reality

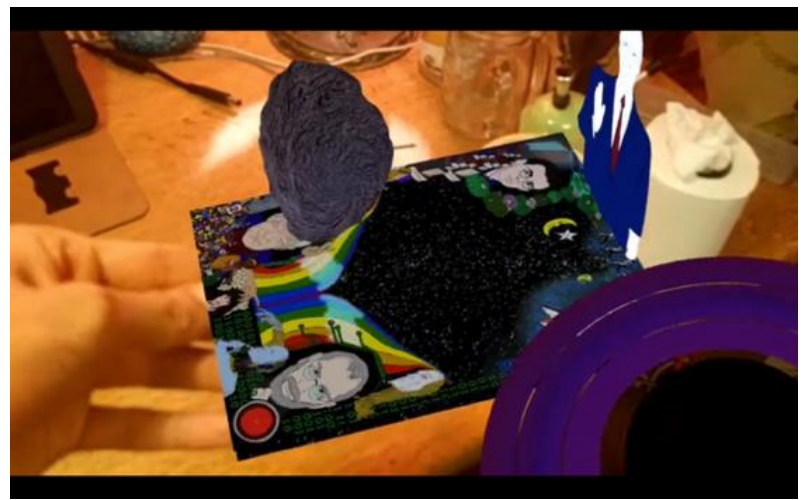

Figure 4: MR - mixing current reality with Cyberland. 


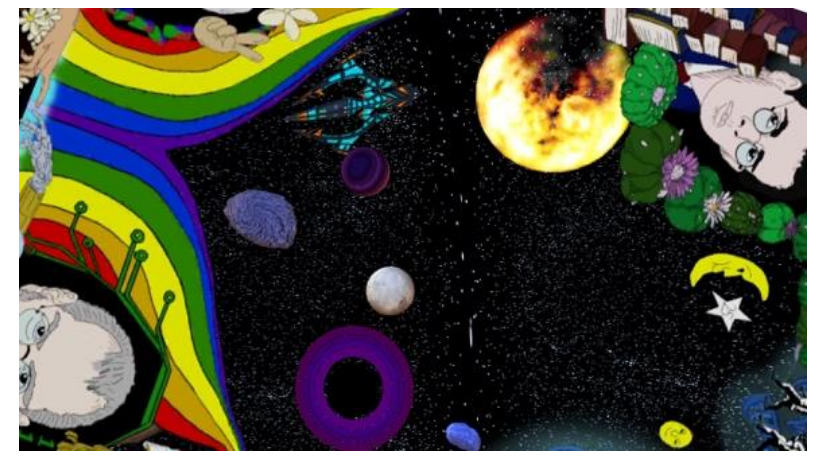

Figure 5: VR - entering into the cyberland and exploring the adventure of the painting. See also online: https://vimeo.com/251523653 (password: OSC).

\subsection{Context Engineering}

Adventures in Cyberland engineers the context of traditional way of reading a book, story telling and exploring a painting by providing new forms of experiences between the body of art and the audience.

Instead of reading the story of a character, page by page, Adventures in Cyberland provides the opportunity for the experiencer to become the main character of the story. Our 'Aliceers' can choose the story they want to interact with, and they can alter the immersion level of the experience by changing between AR, MR, and VR, and learn by interacting with the world beyond the paintings in the book.

The term 'context engineering' comes from 'Contextology', as discussed by Carl H. Smith (2016). 'Contextology' is the study of context or the science of 'Context Engineering' (CE). CE focuses less on engineering content and instead attempts to manipulate and create context directly. This is achieved where we are enabled (via CE tools) to reconfigure our own perception and cognitive abilities directly (individually or in groups) as the primary 'content'. This means that the lenses through which we experience the world are becoming more adjustable than ever. CE considers the broad spectrum of what augmentation can do but also highlights the dangers of focusing solely on technology. CE gives us new abilities, control over our senses and the ability to develop new forms of perception, providing us with a new type of self and societal exploration. Smith suggests that since our society has already produced enough content, now it is time to engineer these into new possibilities and open new doors of perception.

\subsection{Nostalgia}

We are actually currently going through a time of context engineering. For example, Star Wars recently launched their XR experience "Secrets of the Empire" where the audience not only experiences a Star Wars story, but can also step into it an become one of the characters (London 2017). Such projects tap into a demand and longing for nostalgia, while also reflecting how as our civilisation is collectively evolving, so too are our stories and the ways of experiencing them.

As tool-making creatures, we shape tools and there after they shape us and our understanding of reality. The tools that we are exploring with currently have brought possibilities to create alternative realities, in which we can collectively grow, evolve and explore the deepest secrets of the imagination. Thus, through the use of these tools, the time to join Alice and have enjoyment in this 'context engineered' Wonderland has come in form of Adventures in Cyberland. Adventures in Cyberland uses nostalgic elements from Lewis Carroll's original Wonderland, and provides a decentralised educational playground as a platform both for children and adults to learn through exploration.

Adventures in Cyberland provides one of the earliest versions of a portal into Cyberland. As a decentralised ecosystem of educational playgrounds, Cyberland and its characters will eventually grow as a platform with the demands of the society, as realised through other projects. Looking forward, the next section discusses these plans in further detail.

\section{CYBERLAND}

Soon Cyber Rabbit will provide a decentralised ecosystem model through which people will be able to collectively decide on the type of characters and stories our societies need. Cyber Rabbit will bring these into Cyberland. For example, different groups of people, or organisations, may get together and want to raise awareness on, for example, unfair censorship on social media, asylum seekers, or current environmental issues. Through the system provided they will be able to contact Cyber Rabbit, and together we will collectively fund and bring these stories and characters into the Cyberland platform. As the interest in Cyberland increases, many different stories pointing at different topics will come into existence.

This ecosystem will give us the opportunity to see our society from a holistic perspective, and develop empathy for one and another as we experience each one of these stories. On the other hand, this platform also gives us the chance to provide our children with content that is created collectively. This will improve interconnectivity within society. This is very crucial for our times because, how can we try to imagine peaceful futures when our upcoming generations are growing in an 
environment that normalises ideas of fear and hatred? Current stories force-feed our young ones with insipidly paranoid scenarios that keep our fear reflexes chronically throbbing. War, violence, sexism, racism, stereotypes and any other imaginable forms of separations are being projected on all of us through films, super heroes, games, and now even AR, MR and VR experiences.

Because of the normalisation of different forms of the fear-inducing scenarios through the media, we now even have a society that is fearful of getting trapped in a Cyber World. Yes, this is a possibility - but one of many! As a society, we need to understand that if we can believe that these technologies entrap us, then we can collectively believe that they can free us from the separation that is created by the current 'operating system'. In response to this, Cyber Rabbit offers Cyberland as a platform where the experiences and characters grow by the demand of the collective through a decentralised system. Current topics that are being built in Cyberland are discussed in the following subsections.

\subsection{Decentralised Society}

Decentralisation is one of the growing interests that is currently being experienced in business models and communities. There is even a growing interest in bringing decentralised living systems to wider society, which is somewhat revolutionary. Decentralised living systems provide an environment where instead of having a control centre, there is collaboration between communities; thus society comes into life through a network of the minds.

One of the first stories in Cyberland will be about a decentralised community that lives on the other side of the book. This story provides an experience where the 'Aliceers' may feel how it could be living in a decentralised society.

At the moment, Cyber Rabbit is in contact with Satalia who provide expertise in the Artificial Intelligence (AI) industry. Satalia is one of London's leading Decentralised Al companies. The company's aim is to provide opportunities and solutions with Al that enable everyone to do the work they love. For more information, please see the founder of Satalia's (Daniel Hulme's) ideas on $\mathrm{Al}$ and our collective future (London Futurists 2017).

\subsection{Artificial Intelligence}

$\mathrm{Al}$ is one of the most exciting peaks that our society reached so far. We are now becoming able to bring new forms of intelligence into existence. The image of Artificial Intelligence has been somewhat overshadowed by fear-inducing scenarios where robots take control over humans or they decide to kill all of us. Yet it holds great potential.

In Cyberland our 'Aliceers' are invited to explore what we could actually achieve if we collectively and lovingly crafted these artificial intelligence systems with intention to help and understand one and another. We will be exploring some of the ideas of Ben Goertzal and his company, SingularityNET. This company works on providing a decentralised $\mathrm{Al}$ system that aims to provide a 'super intelligence'. We are also interested in Ray Kurzweil's idea of the 'Age of the Spiritual Machines', and the 'Technological Singularity' of Murray Shanahan.

\subsection{Crypto Currency}

The status quo that still tries to control our society is currently experiencing one of the biggest challenges in its entire history of domination, through the Internet. In only 20 years, the Internet has allowed people to reconnected and establish new concepts of 'trust', creating new online communities and value systems.

Until today, one of the greatest control tools of the current dominant 'operating system' has been money, which is controlled by a small portion of the whole society. Today this unfair tool is facing a significant challenge, as people are moving into using cryptocurrencies that are built through trust in one and other (KR1 2018).

Cyber Rabbit is in contact with expertise from the Crypto Currency world for both writing the educational stories for Cyberland and building the Cyberland's ICO coin system. In this way, Adventures in Cyberland will raise awareness of Crypto Currencies, what they mean, how to use them. We will soon be launching the ICO tokens of this platform, which may be used to suggest characters, stories and fund them to bring into existence.

As this is a decentralised platform, the experiencer (the 'Aliceer') will also have the option to choose contribute towards the building of this platform. It could be that they go into Cyberland and build stories with Virtual Tools, animate the characters and develop new experiences. This will give the chance of earning ICO coins for their contribution in building the ecosystem.

\subsection{The Internet}

It's been only 20 years since the World Wide Web connected the world and only 10 years since smart devices entered general use. Our society now has 
become ultra hyper-connected and the Internet has become the most powerful tool that is already causing major shifts in our society, structures, and our lives. Yet, we have already begun to take this for granted as it merged into our daily lives.

One of the stories provided in Adventures in Cyberland explores how the Internet has sped up the evolution process of our society. Children born nowadays cannot distinguish the difference between the world before the Internet and after. Therefore, it is important to raise awareness on how the Internet changed our society's structures from its early days onwards.

\subsection{Evolution of consciousness ....and where we are going as species}

In this part, the stories and the characters will be exploring our evolution process, how technological revolution is affecting our evolution, and how the rise of the cyber culture will shift and empower the creative potential of individuals. I discuss these ideas in more detail in my talk 'Evolution of Consciousness in the rise of the Cyber culture' (Cyber Rabbit 2018).

\subsection{Extension of reachable realities}

In the early $21^{\text {st }}$ century, current civilisation has begun creating new realities, thus we have started extending our reachable reality possibilities. Only in around 40 years, our computer graphics moved from sticks shooting pixels to each other to photorealistic computer-generated human-looking characters.

See the game Pong (Figure 6) from the 1970s and a recreated version of Princess Leia from "A New Hope" (1997) in "Rogue One" (2016) (Figure 7). If this is what we have achieved in only 40 years of development of the computer generated graphics, then what we can create together with what Extended Reality technologies are providing us is an unbelievably exciting thing that allows us to open many more new doors of perception.

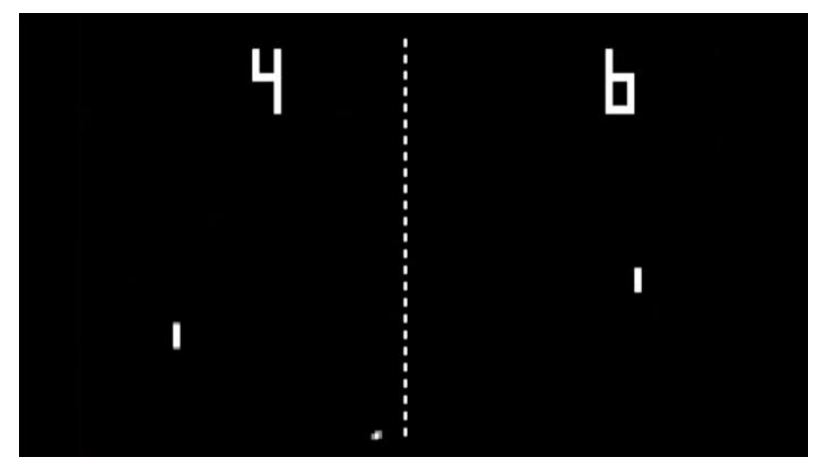

Figure 6: Pong video game.

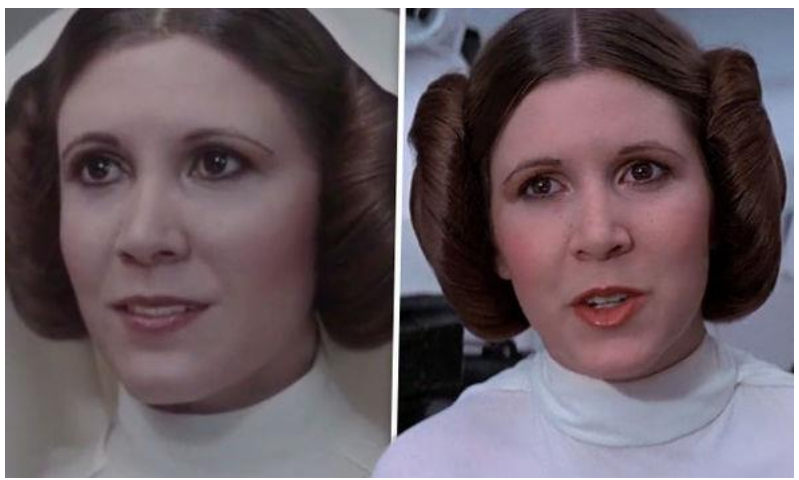

Figure 7: Princess Leia.

Through both through the Cyber Mushroom experience itself and the characters in the Adventures in Cyberland, the audience goes on a journey, exploring the possibilities that these tools are providing us.

\subsection{Cyberdelics}

Cyberdelics are the experiences through which we may incubate and explore what we have learnt from the psychedelic experience in order to design and evolve a whole new range of altered states of consciousness experiences created by using extended reality technologies.

There are already collectives, companies and institutions exploring these concepts: Cyber Rabbit, Virtual Awakening, Psych-Fi, and Contextology. These real-life groups will be present in Cyberland as communities with their characters and experiences so people can go and explore their newest content, experience, events. For more information on these groups, see also the following websites:

- $\quad$ http://www.cyberrabbit.co.uk

- http://www.cyberdelicsincubator.com

- http://www.virtual-awakening.com

- http://www.psychfi.com

\subsection{Empathy Tools}

Here we explore the potential of XR technologies in creating empathy tools to improve our understanding of one another.

\subsection{Characters of Cyberland}

Beings of Cyberland will come into existence through the collective demand and exploration. Therefore the character designs will also grow through this collaboration. The aim is to create characters through that different areas of society can find familiarity with, and be able to relate to. 


\section{CALL FOR ACTION}

While Cyberland is being built, Cyber Rabbit will be on the street spreading the 'word of mouth' about this upcoming collective education playground, and will be inviting people to contribute into building Cyberland collectively.

This will initial be spread around through a Cyber Rabbit XR experience that is given to people for free. This will be a postcard that has the image of Cyber Rabbit meditating, which will be used as a trigger image. The given person downloads the Cyber Mushroom experience and wakes up the Cyber Rabbit from meditation.

Once awakened, Cyber Rabbit will be talking to the experiencer (the 'Aliceer') and the viewer will be able to interact through virtual buttons. Later, Cyber Rabbit offers a deeper experience through the Cyber Mushroom where the experience falls from $A R$ and MR into VR. In the Cyber Rabbit VR experience, the audience will find themselves going through many different stories - from the rise of cyber culture to the evolution of consciousness. They may also explore the idea of collectively building a realm where we bring super heroes into existence, explore our curiosities, and share our thoughts.

Cyber Rabbit will welcome the audience to Cyberland and invite them to take action. The experiencer will then come back to our ordinary reality and they will be asked to share their feedback on how they felt by following Cyber Rabbit. They will then be directed to the online communication platforms such as websites and social network platforms to follow and take action.

Cyber Mushroom is already ready and the Cyber Rabbit experience is already underway. It will be ready take to the streets as soon as end of May, and over the summer. The experience will also give some Cyber Mushroom coin samples to people to spend on the website, to recommend ideas to Cyber Rabbit to bring into existence.

We are working on bringing this experience into existence as we believe in our vision. By freely sharing this Cyber Rabbit experience, we are sharing the ability and excitement of creating immersion that empowers the imagination through cyberspace, and inviting people to take action by sharing their ideas by using their Cyber Mushroom Tokens. This will give us the chance to encourage people to take full action, share their ideas and allow us to see what aspects people want to change in society. So then, once Cyberland receives enough demand for 3-5 characters and story options, a new pool will come into existence for people to re-vote on these ideas. At this point, we would like to make one thing clear: these collectively created concepts will not be in competition. Each one of them will eventually come into existence. Each of them will have a Cyber Mushroom cap to bring together. Once this is achieved, Cyber Rabbit take action to bring the characters and stories into existence, with the funds collected from Cyber Mushroom sales being used to fund these projects.

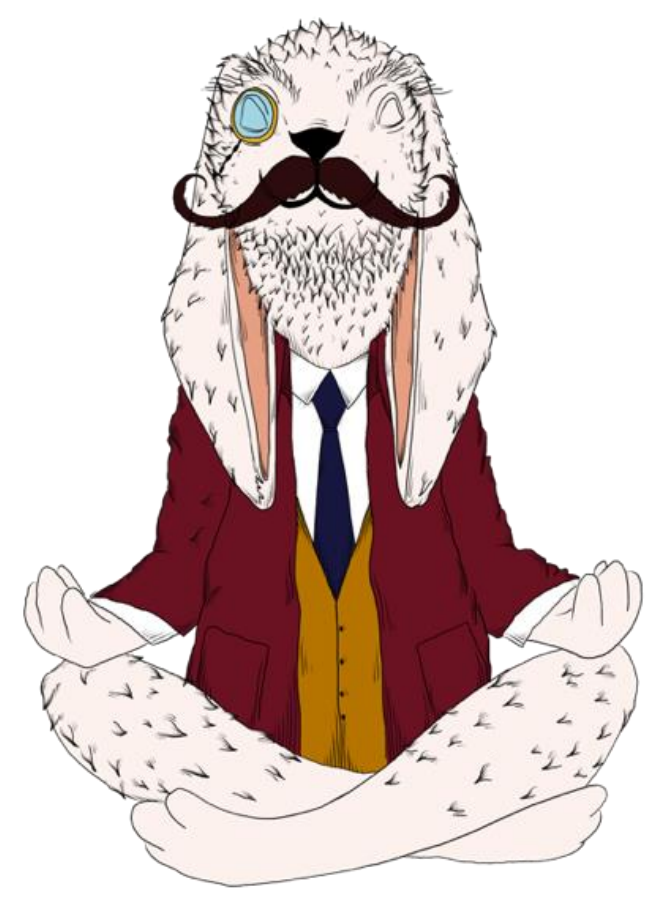

Figure 8: Cyber Rabbit meditation illustration.

\section{REFERENCES}

Ariel, G. and McKenzie, S. (2017) Augmenting Alice. Amsterdam, BIS Publishers.

Breaking Convention (2017) Carl H Smith Boundaries And Application Areas Of Perceptual Technologies To Create Non-Drug ACSs [video]. https://www.youtube.com/watch?v=YfR2Lh6BLhg (retrieved 12 February 2018).

Carroll, L. (2013) Alice's Adventures in Wonderland. Dover Publications.

CiscoFrance (2017) The Future of Al: from Deep Learning to Deep Understanding, Ben Goertzel [video].

https://www.youtube.com/watch?v=nbUg9luls 8 (retrieved 2 February 2018).

ConsenSysMedia (2017) Dr. Ben Goertzel \& Sophia The Robot - Artificial Intelligence, Robotics \& Blockchain [video]. https://www.youtube.com/watch?v=1rrBBfVBx2Q (retrieved 4 February 2018).

Cyber Rabbit (2018) Evolution of Consciousness in the Rise of Cyber Culture by Batuhan Bintas - 
CyberRabbit [video]. https://youtu.be/Q6Ye7N3AJE (retrieved 27 April 2018).

Decentraland (2017) Decentraland a virtual world on open standards [online].

https://decentraland.org (retrieved 9 March 2018).

KR1 (2018) Owen Barnes- KEYNOTE Pathfounder Crypto Economics Conference [video]. https://www.youtube.com/watch?v=zk1xUUz4uxA\& feature=youtu.be (retrieved 14 Apr 2018).

Kurzweil, R. (2000) The Age of Spiritual Machines. New York, Penguin.

London, A. (2017) Free-roaming Star Wars VR Experience hits London's Westfield this Winter [online]. https://www.techradar.com/news/freeroaming-star-wars-vr-experience-hits-londonswestfield-this-winter (retrieved 9 January 2018).

London Futurists (2017) Al and our Future, with Daniel Hulme [video].

https://www.youtube.com/watch?v=XUIJ5ZmrLig (retrieved 12 August 2017).
Science, Technology \& the Future (2017) Ben Goertzel: AGI will obsolete human life as we know it [video].

https://www.youtube.com/watch?v=qQvoVzDt2yk (retrieved 14 April 2018).

Shanahan, M. (2015) The Technological Singularity. Cambridge, MA, MIT Press.

SingularityNET (2018) SingularitynNet [website]. https://singularitynet.io (retrieved 11 April 2018).

Smith, C. H. (2016) Contextology - The Science of Context Engineering by $\mathrm{Dr}$ Carl Smith [online]. https://wiki.mq.edu.au/pages/viewpage.action?pag eld=226394118 (retrieved 6 February 2018).

Virtual Futures (2017) Context Engineering | Carl $\mathrm{H}$. Smith | Virtual Futures Salon [video]. https://www.youtube.com/watch?v=a aybdzz6ml (retrieved 14 September 2017).

Weinel, J. (2018) Inner Sound: Altered States of Consciousness in Electronic Music and AudioVisual Media. New York, Oxford University Press. 